\title{
TEamWORK AND COLLABORATION IN COGNITIVE Wireless NetWorks
}

\author{
Keith E. Nolan and Linda E. Doyle, CTVR, Trinity College, Dublin
}

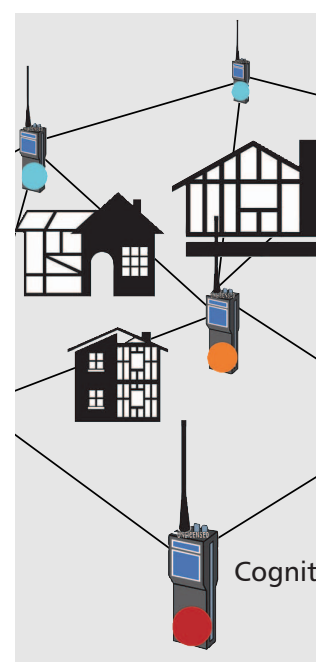

The authors discuss the principles and significant potential of teamwork in cognitive networks. These concepts represent a new evolutionary stage in the development of cognitive radio and cognitive networks.

\section{ABSTRACT}

This article looks at the principles and significant potential of teamwork in cognitive networks. These concepts represent a new evolutionary stage in the development of cognitive radio and cognitive networks, where wireless communication progresses from an individual, device-centric approach toward group and team behavior. This creates the potential for more effective and more robust communication solutions when deemed necessary. The key elements of effective cognitive network teamwork are introduced in this article. These include group formation, distributed co-ordination, goal and role identification, accountability, and reward mechanisms for the outcomes of team behavior. The value of the group as a whole can be increased rather than individual gain for only a single device. Focusing on an early experimental cognitive network teamwork testbed designed by CTVR, this article outlines the potential of developing cognitive networks that can work as a team. Potential applications and market opportunities for this technology also are described.

\section{INTRODUCTION}

The principles of effective teamwork, applied in a wireless communications context, can offer more robust and effective communication for some challenging and emerging scenarios involving networks of distributed wireless nodes. Teamwork and collaborative approaches can be used in cases where an individual entity does not have the resources, the capacity, or the time to solve a problem on its own. In addition, a distributed teamwork approach potentially can help counteract the inherent volatility of wireless networks. The concepts discussed in this article build on the principles of grid computing and the collaborative and cooperative behavior of networks to look ahead to the future potential of wireless networks. Therefore, teamwork represents a potential new evolutionary stage of cog-

This material is based upon work supported by Science Foundation Ireland under Grant no. 03/CE3/I405 as part of the Centre for Telecommunications Value-Chain Research (CTVR) at Trinity College Dublin, Ireland. nitive radio and cognitive network development. The first stage in exploiting this emerging capability is to gain an understanding of the underlying principles of effective teamwork in a cognitive network context.

In the following section the concept of a cognitive network is described. Next, an introduction to the concepts of teamwork and collaboration is presented. We then describe an early experimental scenario outlining the value of teamwork in a heterogeneous ad hoc network, where the primary objective of the team is to facilitate communication for a group of users. The more forward-looking potential value of cognitive network teamwork is outlined, and we conclude in the final section.

\section{COGNITIVE RADIO AND COGNITIVE NetWORKS}

For the purposes of this article, it is useful first to clarify what the authors mean by the term cognitive radio.

A cognitive radio is a wireless communication system that can acquire and use information relating to the state and resources of the device and information relating to the context in which the device is operating to help achieve designated objectives [1]. Cognitive functionality can influence the operation and structure of the entire communications stack and subsystems. Therefore, the term cognitive radio more accurately can be described in terms of a complete system or as a node. A cognitive node uses contextual information to devise and implement an information-conveyance solution. Contextual information can be derived from the physical (node state and device resources, spatial, environmental, network topology, and communications channel information), regulatory and policy (frequency spectrum usage rules and etiquettes, licenses, user access rights), social (individualistic or collaborative and distributed network existence), and economic (payment mechanisms, spectrum market environment) domains.

A cognitive network is a network of nodes with cognitive functionality [2]. This network can be formed and evolve on an ad hoc basis, and each of the nodes in the network may be required to contend with different fixed and 
time-varying constraints. These can include (and are not limited to) the available energy, cognitive abilities, and radio frequency (RF) front-end capabilities.

Cognitive functionality also can be leveraged through the combination of resources and individual cognitive abilities of the nodes in a distributed network. This is similar to the concept of grid computing [3, 4], where distributed computers are employed when required, to solve problems that may not be feasible to solve by a single computer operating on its own. Each of the computing nodes in a grid may have a predetermined task and be configured to perform a fixed set of tasks. By combining cognitive functionality and an advanced form of the principles behind grid computing, where each node can learn, change its role in the network, and handle new tasks required to solve emerging problems, the potential for new and enhanced wireless communications applications increases. The ability to learn, make decisions, and formulate conclusions are key elements of a cognitive node [1]. By introducing the ability to reconfigure in the application, structural and parameter planes into this pool of capabilities, a reconfigurable cognitive node can be created.

Collaborative sensing is a related research topic that uses a network of distributed nodes to solve problems or collect information. Zhao et al. [5, 6] describe a sensor network of distributed energyconstrained nodes whose main objective is to track the movement of an entity. The ability to collaborate is required to help accomplish this goal.

\section{PRINCIPLES OF TEAMWORK}

A team can be viewed as a group participating in a consensual form of intentional collaboration with each other. Collaboration can involve entities coming together to work on a single project, whereas an effective team can stay together to work on additional problems and can evolve further with experience. A team effort can significantly increase the chance of a successful outcome when faced with a challenging problem. In essence, teams consist of a relatively small number of individuals with complementary skills and resources, who work together for a common purpose or toward a common goal or set of goals [7]. This team also is accountable for its actions. Knowledge and additional skills that may be gained from repeated group activities also can help each member of this group to evolve. The formation of a successful team helps increase the group knowledge base creating a pool of resources and wisdom.

To create a team, the establishment and maintenance of a team of entities (or nodes in the context of this article), is a key requirement. In addition, the ability to identify potential team members, form a team, identify and assign roles within that team, establish working practices, and agree on a set of actions are required features. To establish and manage an effective team, an ability to co-ordinate with the leader and team members is vital. Accountability for the action of the team and its members is required to help this team evolve and increase in effectiveness. Accountability in the context of a

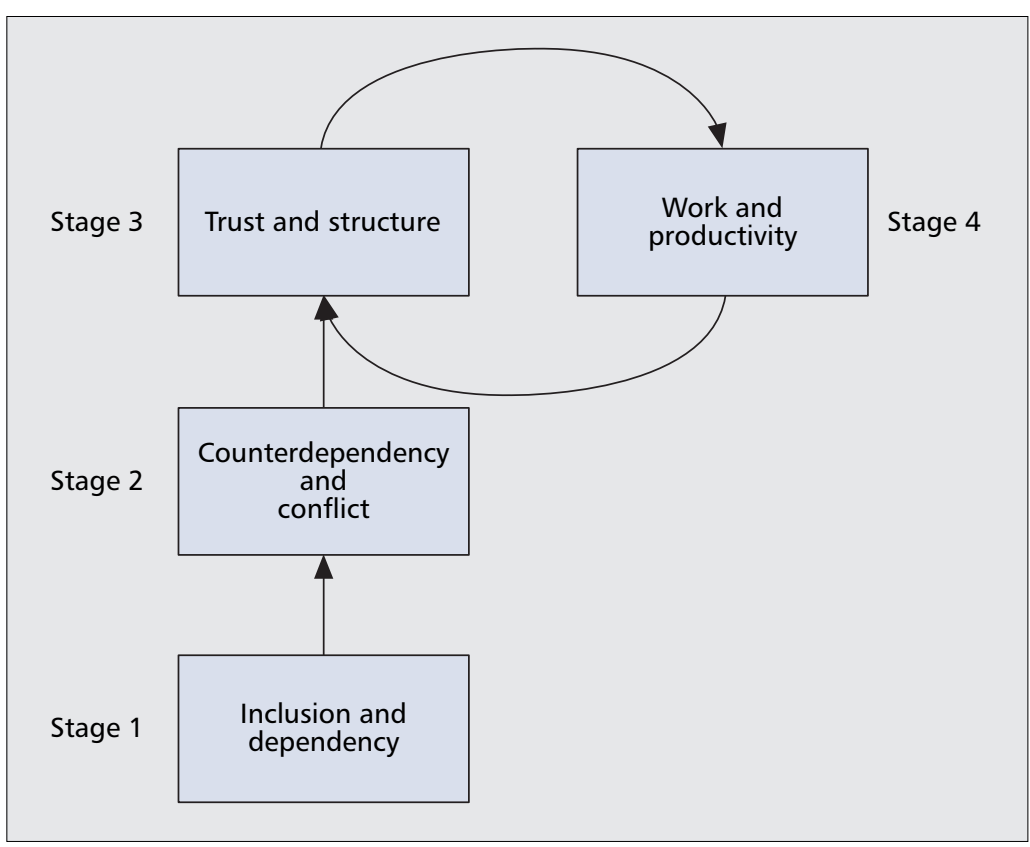

Figure 1. The four stages of team development. A team may alternate between stages 3 and 4 as the network and tasks change over time.

cognitive network team entails determining whether the set of actions performed by the team members and overall outcome of the collective action justified the energy expended. In addition, this involves estimating whether a successful outcome could have been achieved in a more effective or timely manner. This process is important for establishing the value of the decisions and conclusions made by the team.

The value of decisions and conclusions diminishes if they cannot be exploited within a specific deadline. It is feasible that all of the required information may not be available, may require excessive energy or processes to acquire, or there simply may not be enough time to collect and process this information. There also may be a significant time lag as information diffuses through a distributed network. In this case, a fast and frugal heuristic approach can be employed, where a sub-optimal yet sufficient decision can potentially be made using less effort and with some but not all of the required information.

\section{Stages of Team Development}

An effective and productive team does not emerge fully formed, fully informed, and highly productive. In fact, newly created teams progress through a number of stages of development, illustrated in Fig. 1. Conflict, confusion, and communication are three common features of this development path. Four distinct stages can be identified in this cycle [7]:

1)Inclusion and dependency (forming)

2)Counterdependency and conflict (storming)

3)Trust and structure (norming)

4)Work and productivity (performing)

The first stage of team development (inclusion and dependency) involves the discovery and recruitment of suitable and willing candidate nodes to from a group. This also is referred to as the forming stage where a group is formed, but 
In an ad hoc

network, change

occurs as nodes join

and leave the

network. The

structure of a

cognitive network

team also may

change due to the

time-varying

characteristics and

states of each

member node. the member nodes are not yet delegated specific roles. All cognitive radio devices (nodes) within a network are members of this core group. The designated leader in this case is the node initiating the group formation action. At this stage, group members are aware only of the group itself. The purpose, goals, or operational guidelines associated with this body have not yet been communicated to the group members. Conflicts may not yet be apparent as member nodes may be keen to be involved in the emerging group due to the prospect of a teamwork reward or incentive system.

Stage 2, or storming, involves the establishment of a set of team goals and operational guidelines. The clarification of unclear or ambiguous goals also is accomplished during this stage. Conflict may arise when a group goal contravenes the individual goal of a group member. Indeed, group members can attempt to position themselves in a role that is perceived to yield maximum benefit based on their current resources and cognitive reasoning capabilities. Therefore, conflict resolution mechanisms are key to the survival of the group and to help the group to progress to the next stage of team development. The initial high dependence of the nodes on the group leader reduces as the team develops.

In stage 3 of the team development process, or the norming stage, trust relationships between the member nodes develop. With a clear and agreed set of goals, the focus of the group moves toward identifying the strategies, techniques, and work practices required to attain the desired goals. The team become more defined as redundant or unwilling nodes are bypassed. As the team becomes more focused on the tasks in hand, the role of the leader evolves from a director to a consultatory role.

Stage 4 is classified as the performing stage, where team members have a clear idea of the team goals and their individual roles and begin to work on their tasks. The team itself contains the smallest number of members required to attain the desired goals, and assigned roles match the skills and expertise of each member. These roles may be assigned based on the RF front-end specifications of the member, on cognitive abilities, and on the radio range proximity to other team members. Subgroups tackling specialized subtasks may form, and this activity can result in an important contribution to the overall effectiveness of the team.

In an ad hoc network, change occurs as nodes join and leave the network. The structure of a cognitive network team also may change due to the time-varying characteristics and states of each member node. As a result, a team may alternate between stage 3 (trust and structure) and stage 4 (performing) as change occurs, as illustrated in Fig. 1. Three main approaches can be taken to handle this change within a cognitive network team:

- No action: accept the loss of a team member and the extra time that may be required to accomplish the team goals as a result. The outcomes of the ongoing cognitive network innovation processes may actually mean this loss does not affect the overall effectiveness of the team.
- Reconfiguration: adjacent (and capable) nodes may attempt to augment their own list of actionable items to share/absorb the role(s) of the expired or unresponsive team member.

- Supplantation/recruitment: an expired or unresponsive node is either supplanted or replaced by a new node. The team may evolve to the second or third stage of the team development cycle due to possible conflicts as existing team members adapt to the change in workload and team structure.

\section{TEAM ROLES}

As explained at the beginning of this section, an effective team includes members with complementary abilities and resources. Members of this team can adopt different roles based on the capabilities and resources of each node member. These roles also can change over time. Belbin [8] and Wheelan [7] describe these team roles in a human context and discuss how these team roles can consist of either one, or a combination of, the following four general categories:

- Diverger

- Assimilator

- Converger

- Accommodator

A diverger is oriented toward feelings and people. In a cognitive radio context, the diverger aims to maximize benefits for the network and individual nodes. The initiator of a cognitive network team is the initial candidate for this role; however, this role can shift between nodes. The reason for this is that initial candidacy for this role is not an indication of long-term suitability due to the changing states and time-varying nature of the resources available to the node.

An assimilator is oriented toward rational thought and logical analysis of a situation. A cognitive node with decision making and conclusion formation abilities can adopt this role. An assimilator can make use of internal and external observations, knowledge of previous outcomes, and conclusions as part of logical analysis process. The assimilator also can influence how processing loads may be redistributed among the team as the abilities, available resources, and topology of the network changes. This may be required to help solve emerging problems by taking preemptive action.

A converger is oriented toward constructive action and practical implementation, converting the team-plan and viable conclusions into a set of actionable items. The ability of a cognitive node to reconfigure, directed by the outcomes of the assimilator is an example of this role in practice.

The final key role is the accommodator, oriented toward exploration of new techniques and includes risk taking. In a cognitive network context, an accommodator is a node with strong learning and hypothesis testing abilities. An additional characteristic is that the node has sufficient energy to distribute the outcomes of these activities to the other team members. The main output of this entity is the development of new conclusions based on historical stimuli, action, and conclusions. Innovation and creativity are also characteristics of this role, where new solutions to team challenges that may be in a differ- 
ent direction than the team's current direction of collective thought are developed.

\section{INITIAL TEAMWORK EXPERIMENTATION}

One of the early gains associated with teamwork is the ability to facilitate communication that would not otherwise be possible if all the nodes adopted an individualistic approach. This section examines a simple scenario, building on the concepts explored in this article to demonstrate the value of teamwork and indicate the forwardlooking potential of this concept. The objective of the team is to enable nodes in the ad hoc network to communicate with an access point and thus serve the communication requirements of the users. The key performance measure of teamwork in this initial scenario is whether this objective can be achieved.

\section{EXPERIMENTAL SCENARIO: FACILITATINg COMMUNICATION}

Consider the network in Fig. 2a. This depicts an ad hoc network that is operating in the $2.4 \mathrm{GHz}$ industrial, scientific, and medical (ISM) band for local communication among nodes. The nodes denoted by the small circles are simple ad hoc nodes, serving a test group of users that can communicate only using single-carrier modulation (differential binary phase-shift keying) and very low power (less than $10 \mathrm{~mW}$ ). The nodes denoted by the larger circles are nodes with cognitive functionality and frequency agile frontends that facilitate communication in both this ISM band and other frequency bands.

In an ad hoc network, very often there is a node in the network that connects to an access point (or acts as a bridging node between two contiguous networks) and thus facilitates communication between the ad hoc network and the wider world. We are interested in the case in which the access point does not work on a fixed frequency but instead is an opportunistic user that avails of white spaces that occur in the spectrum of a primary user. Therefore, the frequency used either to communicate with the access point or to the bridging node of the second network may change. The ISM-band user nodes of the network cannot connect to the access point on their own, but the highly functional cognitive node can.

Our initial experimental testbed, depicted in Fig. 2b, consists of one access point node and an ad hoc network of three nodes, consisting of two user nodes and one highly functional cognitive node. The cognitive node in the ad hoc network acts as the bridging node between the ad hoc network and the access point. In addition to ISM band capability, the cognitive node is capable of communicating with the access point within a 25 $\mathrm{MHz}$ bandwidth spectrum segment centered at $2.35 \mathrm{GHz}$, licensed by the Commission for Communications Regulation (ComReg) for cognitive radio testing by the Centre for Telecommunications Value-Chain Research (CTVR). Incumbents with primary access to this band must be avoided when present; thus, a fixed common frequency between the access point and the cognitive node in the ad hoc network cannot be agreed upon in advance. Therefore, the team consists of

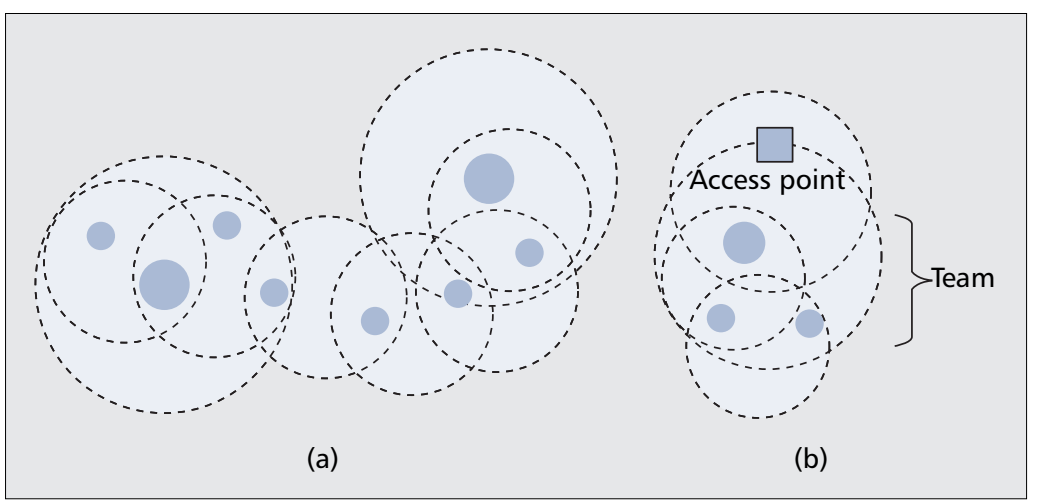

Figure 2. An illustration of (a) a heterogeneous ad hoc network; (b) the initial experimental testbed configuration used by CTVR.

these heterogeneous nodes with complementary abilities and resources, as illustrated in Fig. 2 b. The common objective among this team is to serve the communications requirements of the users. The secondary individual and pre-determined objectives of the simple ISM band nodes is to maximize their operating lifetimes, which they can do by availing of the cognitive node rather than expending excessive energy attempting to connect to the access point that is out of range of the simple ad hoc network nodes.

For simplicity, we illustrate a scenario in which the access point uses orthogonal frequency-division multiplexing (OFDM) and must change the frequency of operation as white space spectrum changes due to incumbents appearing on channels within this frequency range (where a vector signal generator assumes the role of an incumbent in the experimental testbed). The access point uses a form of digital watermarking known as embedded cyclostationary signatures $[9,10]$. Rather than relying on an energy-detection technique to identify the presence of a signal, unique features are embedded in the transmitted signal that the cognitive nodes in the network can identify as the access point. Using the digital watermarking as an aid, the highly functional cognitive node in the ad hoc network can obtain an estimate of the center frequency used by the access point, and establish communications with or rendezvous with the access point. Following this, the node can commence communications and relay information to/from the other nodes in the network.

In this scenario, both the cognitive access point and cognitive node in the ad hoc network use universal software radio peripheral (USRP) RF hardware designed by Ettus Research (ettus.com) with CTVR-designed software completing the rest of the transceiver signal chain. The first stage of the teamwork process (forming) involves the cognitive access point transmitting an advertisement signal, or beacon, on an unoccupied channel in the CTVR test spectrum centered at $2.35 \mathrm{GHz}$. This transmitted signal has a bandwidth of $1 \mathrm{MHz}$. In this experiment, it is generated using a 256-bin inverse fast Fourier transform (IFFT), of which 189 subcarriers are used for data from the nodes, and eight subcarriers are pilots used for fine frequency correction and frequency domain equalization at the receivers. The zero frequency (DC) subcarrier is not used, and 


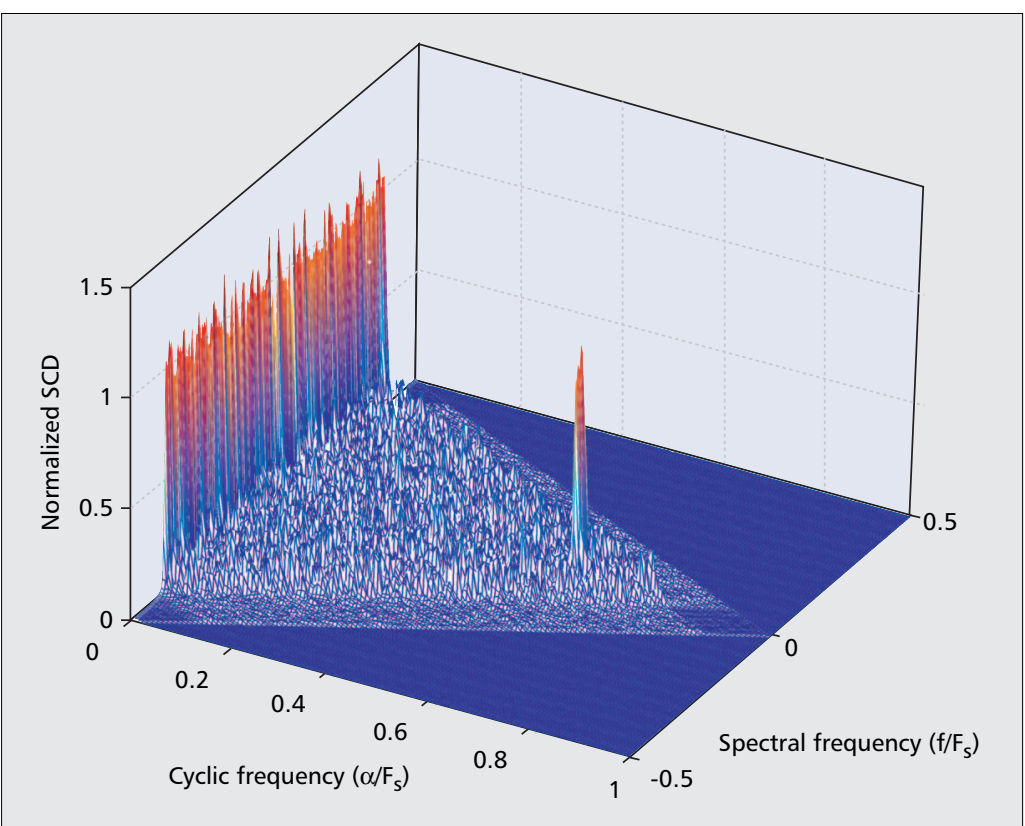

Figure 3. A plot of the spectral correlation density (SCD) showing a distinct peak corresponding to the embedded cyclostationary watermark at $\alpha / \mathrm{F}_{\mathrm{S}}=$ 0.65 .
- Cognitive node: Establish and maintain connectivity with the access point and conveys information to/from the user nodes to this access point (diverger/accommodator).

This is a relatively simple scenario, without sophisticated teamwork processes and complex counterdependency and trust issues. Therefore, progression through the storming and norming stages is rapid. As a result, the team evolves quickly to the performing stage where communications traffic from the connected nodes in the ad hoc network can transfer to and from the cognitive access point. In this case, the cognitive access point initiates the team formation and therefore, assumes the role of diverger. Then, this role is taken over by the cognitive node in the ad hoc network as it focuses on maximizing the benefit of team action for the entire network. It achieves this by ensuring that the user nodes can both establish and maintain the communications link with the access point.

The principle gain of this scenario is that communication between the simple user nodes and the cognitive access point can be achieved where it was not possible without teamwork. Although this is a simple experiment without the need for a significant delegation of tasks to team members or innovation, it nonetheless provides an indication of the value of team action. The potential reward for performing as a team and achieving the primary objective of facilitating the communications requirements of the user group is the collection of revenue. In an extension of this scenario, user devices that can achieve successful communication with the access point through this process of teamwork can effectively rate the cognitive node in terms of performance. The cognitive node also learns from the experience by storing the center frequencies where successful communication with the access point occurred, as a means of attempting to reduce future search and rendezvous time with the access point.

\section{FORWARD-LOOKING POTENTIAL OF COGNitive Network TeamwORK}

Cognitive network teamwork has the potential to play a valuable and interesting role in future wireless communications networks. The market opportunities for this capability include entertainment (in the form of combined real-world and virtual multiplayer games), in-home networks of devices that can combine their capabilities and resources, in addition to military, public safety, biomedical, industrial, and consumer applications.

The value lies in the ability to form a team of cognitive nodes that can attempt to solve a series of different wireless communications challenges, where a single node cannot do so on its own.

In the case of public safety, and where a single wireless device may have a limited operational range, a cognitive network team could help facilitate interoperability between different emergency services over a wide area as they converge on a scene. Building on the principles of collaborative sensing, this team also could act simultaneously as a sensor network to detect desired features (e.g., RF emissions, infrared, pressure, temperature, and air quality). Figure 5 
is an illustration of a distributed network of cognitive nodes deployed over a geographical area. In this figure, each of the nodes has a specific role to play in the network team (indicated using the colored circles). This is to highlight that unlike a collaborative or cooperative network of similar nodes directed by a single entity, a cognitive network team is comprised of nodes with complementary abilities and resources. In addition, although the team formation may be initiated by one entity, the nodes can operate on their own within their assigned roles and tasks.

\section{CONCLUSIONS}

This article introduced the concept of teamwork and collaboration in cognitive networks and described why these are important in the context of future wireless communications. Looking ahead, cognitive network teamwork is a potential enabler for the emergence of new and more robust applications involving wireless communications, sensing, distributed decision-making, and control.

An outline of cognitive radio and networks and a description of the principles of team formation and team development were presented. We described how an effective cognitive network team is a relatively small group of nodes with complementary abilities, expertise, and resources, working together toward a common goal, or set of goals, and that are accountable for their actions. By working as a team, the potential to develop and implement an effective solution can be dramatically increased. An initial experimental scenario was described, highlighting the early potential of teamwork in cognitive networks to facilitate communication, leading to an indication of how this concept can have more extensive value in forward-looking network scenarios and markets.

\section{REFERENCES}

[1] S. Haykin, "Cognitive Radio: Brain-Empowered Wireless Communications," IEEE JSAC, Feb. 2005.

[2] R. W. Thomas, L. A. DaSilva, and A. B. Mackenzie, "Cognitive Networks," DySPAN '05, Nov. 8-11, 2005, pp.352-60.

[3] I. Foster and C. Kesselman, The Grid: Blueprint for a New Computing Infrastructure, Morgan Kaufmann.

[4] F. Berman, A. J. G. Hey, and G. C. Fox, Grid Computing: Making The Global Infrastructure a Reality, Wiley.

[5] F. Zhao et al., "Collaborative Signal and Information Processing: An Information-Directed Approach,", Proc. IEEE, vol. 91, no. 8, Aug. 2003, pp. 1199-1209.

[6] A. Ren and G. Q. Maguire, Jr., "A Smart Network with Active Services for Wireless Context-Aware Multimedia Communications," Wireless Commun. and Sys., 1999 Emerging Tech. Symp., 1999, pp.17.1-.5.

[7] S. A. Wheelan, Creating Effective Teams: A Guide for Members and Leaders, Sage Publications, 2005.

[8] R. Belbin, Team Roles at Work, Butterworth Heinemann, 1993.

[9] W. A. Gardner, Cyclostationarity in Communications and Signal Processing, IEEE Press, 1993.

[10] P. D. Sutton, K. E. Nolan, and L. E. Doyle, "Cyclostationary Signatures in Practical Cognitive Radio Applications" submitted to IEEE JSAC, Cognitive Radio: Theory and Applications.

\section{BIOGRAPHIES}

KEITH E. NOLAN (keithnolan@mee.tcd.ie) received his Ph.D. degree in electronic engineering from Trinity College Dublin in 2005 . He is a research assistant with the Centre for Telecommunications Value-Chain Research (CTVR) at Trinity College Dublin, Ireland. He is engaged in several research activities focusing on cognitive radio, emerging network architectures, and dynamic spectrum access tech-

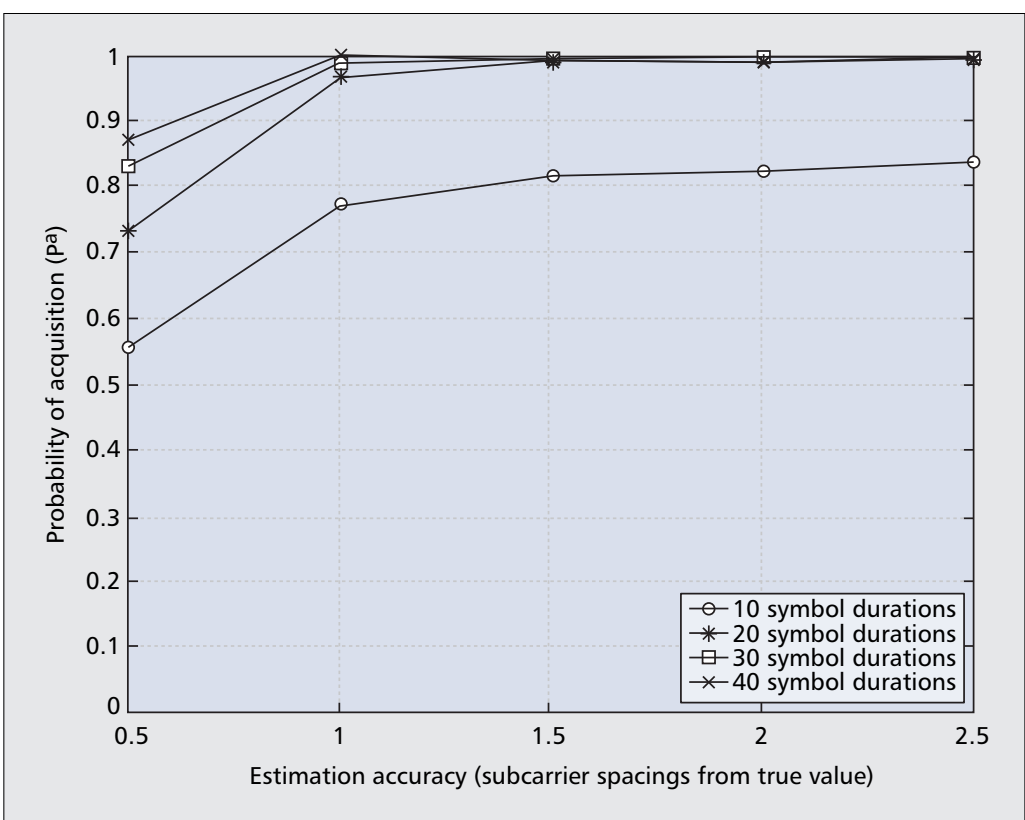

Figure 4. A graph of the probability of detection versus the number of observed OFDM symbols. For an observation time of 40 OFDM symbols, the probability of detection is greater than 95 percent.

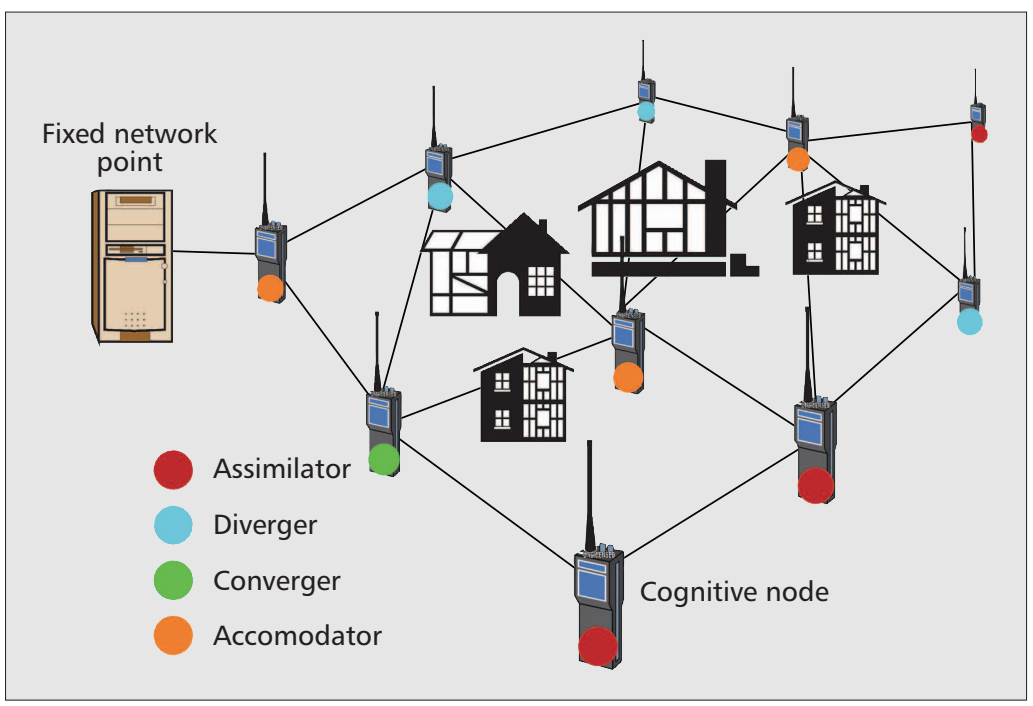

Figure 5. Example of a distributed cognitive network with the roles of accommodator, assimilator, converger and diverger indicated using the red, blue, green and orange circles.

niques. In addition, he also is actively involved in the IEEE Standards Coordinating Committee for Dynamic Spectrum Access Networks (SCC 41) and the Software-Defined Radio Forum cognitive radio working group. He was Demonstrations Chair of the 2007 IEEE Symposium on New Frontiers in Dynamic Spectrum Access Networks (DySPAN) and organized the world's first multi-organization collaborative trials and demonstrations of cognitive radio and dynamic spectrum access technologies that were held in conjunction with DySPAN 2007 during April, 2007 in Dublin, Ireland.

LINDA E. DOYLE (ledoyle@tcd.ie) is a senior lecturer in the Department of Electronic and Electrical Engineering at Trinity College Dublin. She leads the emerging networks strand of CTVR. Emerging networks has a major research focus on reconfigurable networks, cognitive radio, and dynamic spectrum access. She was Deputy Chair of the 2007 IEEE Symposium on New Frontiers in Dynamic Spectrum Access Networks (DySPAN). 